\title{
Open Access Publishing in High-Energy Physics
}

\author{
Salvatore Mele $e^{1,2}$ \\ ${ }^{1} \mathrm{CH}-1211$, Genève 23, Switzerland \\ ${ }^{2}$ On leave of absence from INFN, I-80126, Napoli, Italy \\ e-mail: Salvatore.Mele@cern.ch \\ On behalf of the SCOAP ${ }^{3}$ Working Party
}

\begin{abstract}
The goal of Open Access (OA) is to grant anyone, anywhere and anytime free access to the results of scientific research. The High-Energy Physics (HEP) community has pioneered OA with its "pre-print culture": the mass mailing, first, and the online posting, later, of preliminary versions of its articles. After almost half a century of widespread dissemination of pre-prints, the time is ripe for the HEP community to explore OA publishing. Among other possible models, a sponsoring consortium appears as the most viable option for a transition of HEP peer-reviewed literature to OA. A Sponsoring Consortium for Open Access Publishing in Particle Physics $\left(\mathrm{SCOAP}^{3}\right)$ is proposed as a central body which would remunerate publishers for the peer-review service, effectively replacing the "reader-pays" model of traditional subscriptions with an "author-side" funding. Funding to $\mathrm{SCOAP}^{3}$ would come from HEP funding agencies and library consortia through a re-direction of subscriptions. This model is discussed in details together with a quantitative description of the HEP publishing landscape leading to a practical proposal for a seamless transition of HEP peer-reviewed literature to OA publishing.
\end{abstract}

Keywords: open access publishing; high-energy physics; CERN; SCOAP ${ }^{3}$

\section{Introduction}

The goal of "Open Access" (OA) is to grant anyone, anywhere and anytime, free access to the results of scientific research [1]. The OA debate has gained considerable momentum in recent years across many disciplines, both in the sciences and the humanities. In High Energy Physics (HEP) this debate is driven mostly by two factors:

- The "serials crisis" of ever-rising costs of journals, which has forced libraries to cancel a steadily increasing number of subscriptions, curtailing the access of researchers to scientific literature. This traditional business has become financially unsustainable for libraries and publishers alike;

- The increasing awareness that results of publicly funded research should be made generally available going past the availability of pre-prints, towards peer-reviewed literature.

HEP pioneered OA through its "pre-print culture": the mass mailing for four decades of preliminary versions of articles, so to ensure their largest diffusion. With the onset of the Internet, the HEP community spearheaded the culture of "repositories": online collections of freely accessible pre-prints. Thanks to the speed at which they make results available, repositories have become the lifeblood of HEP scientific information exchange. However, they usually contain the original version of articles submitted to journals, and not the final, peerreviewed, published version. Notwithstanding the success of repositories, there is consensus in the HEP community that high-quality journals still play a pivot role, by providing [2]:

- quality control through the peer-review process;

- a platform for the evaluation and career evolution of scientists;

- a measure of the quality and productivity of research groups and institutes.

A powerful synergy can arise between the strong OA culture of the HEP community, which finds its roots in four decades of preprint circulation, and its continuing need for high-quality journals, leading to a unique opportunity for a possible transition to OA publishing of the HEP peer-reviewed literature. The community is now moving towards such groundbreaking transition through the establishment of a consortium, SCOAP ${ }^{3}$ (Sponsoring Consortium for Open Access Publishing in Particle Physics). This consortium would engage publishers of high-quality peer-reviewed journals in order to cover the costs of the peer-review process with 
funds previously used for journal subscriptions. This idea is viable for the HEP community since the author and the reader communities largely overlap, and are mostly funded by the same actors. This article describes the SCOAP $^{3}$ initiative:

- $\quad$ section 2 puts the HEP publishing landscape into context, and describes the background to OA Publishing in HEP and the steps which led to the $\mathrm{SCOAP}^{3}$ initiative;

- $\quad$ section 3 presents the $\mathrm{SCOAP}^{3}$ model through the roles of the main stakeholders in HEP scientific publishing;

- $\quad$ section 4 illustrates the results of an analysis of the HEP publishing landscape and their consequences on the targets of the $\mathrm{SCOAP}^{3}$ initiative;

- $\quad$ section 5 presents the financial aspects of the $\mathrm{SCOAP}^{3}$ model together with a cost-sharing scenario based on an investigation of the author basis of HEP;

- $\quad$ section 6 concludes the article by presenting the status of the initiative at the time of writing.

\section{Background}

A recent study analyzed articles submitted in 2005 to the arXiv.org repository and classified in the hep-ex, heplat, hep-ph and hep-th categories and subsequently published. Out of a total of about 5'000 articles, more than $80 \%$ appeared in just six peer-reviewed journals from four publishers [3]: Physical Review and Physical Review Letters (published by the American Physical Society), Physics Letters and Nuclear Physics B (Elsevier), Journal of High Energy Physics (SISSA/IOP) and the European Physical Journal (Springer). Almost 90\% of the articles were published by just four publishers, two out of which (American Physical Society and SISSA/IOP) are learned society.

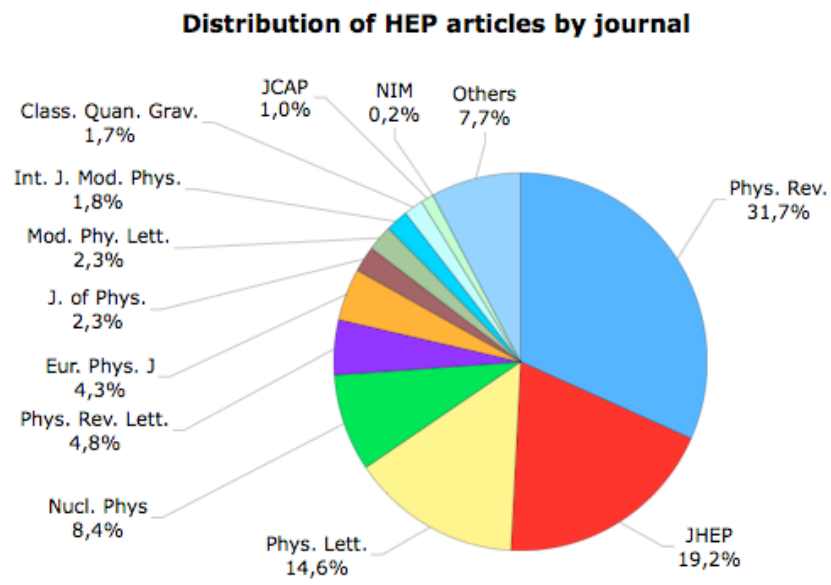

CERN Scientific Information Service

Figure 1: Distribution of the HEP articles submitted in 2005 to the arXiv.org repository under the categories hep-ex, hep-lat, hep-ph and hep-th and subsequently published in peer-reviewed journals. A total sample of about $5^{\prime}, 000$ articles is considered. Only journal with a total share above $1 \%$ are considered, with the exception of Nuclear Instrument and Methods in Physics Research (NIM). The "Others" group comprises 77 remaining journals. From reference [3].

These findings, summarised in figures 1 and 2, spotlight two fundamental points relevant for a possible transition of HEP publishing to OA: the volume of articles is small and these are concentrated in a few core titles, mostly published by learned societies. All HEP leading journals have recently taken a pro-active stance on OA. Journals from the American Physical Society, Elsevier and Springer offer authors an option to pay a fee to make their articles OA, while the Journal of High Energy Physics is recently experimenting with an institutional membership fee. The latter appears a more successful scheme, as funding mechanisms in HEP seldom include overhead for scientific publications to be directly used by authors. Moreover, the direct payment for the OA publication of an articles is perceived in very negative terms by the community, reminiscent of the unpopular "page charges" of some journals. This perception might have extended to other journals, such as the New 
Journal of Physics, which are built on a "pay-per-article" Open Access model, but have so far attracted only a limited HEP content.

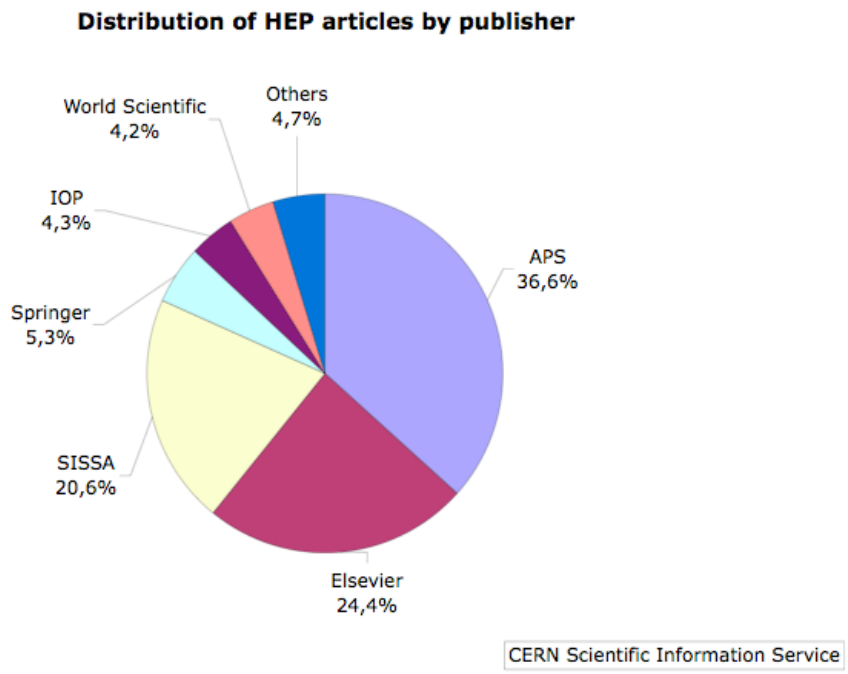

Figure 2: Distribution by publisher of the HEP articles submitted in 2005 to the arXiv.org repository under the categories hep-ex, hep-lat, hep-ph and hep-th and subsequently published in peer-reviewed journals. A total sample of about 5'000 articles is considered. From reference [3].

The debate on OA publishing in HEP was initiated by CERN. CERN is the leading HEP laboratory, with over half a century of history. Its flagship program, the LHC accelerator, will see four large experimental collaborations probe fundamental questions in our understanding of the Universe. CERN epitomizes crossborder collaboration in HEP: the LHC accelerator and detectors include components built in HEP laboratories and Universities around the world; the largest of the LHC experimental collaborations count as many as 2000 scientists, including about 400 students from 160 universities and laboratories spread over 35 countries. As part of its role to chart the future of HEP, in synergy with HEP funding agencies worldwide, CERN promoted several events focussed on OA publishing in HEP:

- September 2005. Open meeting on the changing publishing model. This event brought together representatives of authors, funding agencies and publishers with the aims of first discussing in HEP publishing issues such as publishing costs, competition, fair distribution of costs, opportunities for developing countries, alternative business models and the quality of peer-review [4];

- December 2005. Colloquium on Open Access publishing in particle physics. A representative group of authors, funding agencies and publishers indicated a possible way forward to OA publishing based on three pillars: asserting the complementary roles of repositories and peer-reviewed literature, decoupling preservation issues and the publication model, enshrining the importance of peer-review for evaluation and academic credibility [5];

- December 2005 to June 2006. Task Force on Open Access Publishing in Particle Physics. This tripartite task force composed by authors, funding agencies and publishers was charged by the main stakeholders to "study and develop sustainable business models for OA publishing for existing and new journals and publishers in particle physics". In its report [2] it suggested to establish a sponsoring consortium, SCOAP3, as a central body which would remunerate publishers for the peer-review service, effectively replacing the "reader-pays" model of traditional subscriptions with an "author-side" funding;

- $\quad$ November 2006. Establishing a sponsoring consortium for Open Access publishing in Particle Physics. Following the task-force report and the acceptance of its model by representatives from major European stakeholders, a Working Party was established to develop a specific proposal for the creation of $\mathrm{SCOAP}^{3}$, which is described in this article [6]. More information is contained in the SCOAP ${ }^{3}$ Working Party report [7]. 


\section{The SCOAP ${ }^{3}$ Model}

$\mathrm{SCOAP}^{3}$ will act as a single interface between the main stakeholders of the HEP scientific information market: on one side the author and reader communities and on the other side the publishers of high-quality HEP journals. The aim of $\mathrm{SCOAP}^{3}$ is to establish OA to HEP peer-reviewed articles along the lines of the Budapest Initiative [8], namely "free availability on the public internet, permitting any users to read, download, copy, distribute, print, search, or link to the full texts of these articles, crawl them for indexing, pass them as data to software, or use them for any other lawful purpose, without financial, legal, or technical barriers other than those inseparable from gaining access to the internet itself". At the time of writing, $\mathrm{SCOAP}^{3}$ is an initiative emanating from:

- $\quad$ several European funding agencies, among which IN2P3 and CEA (France), INFN (Italy), MPG (Germany), PPARC (U.K.), and other funding bodies from Greece, Norway, Sweden and Switzerland;

- the two largest European laboratories, CERN and DESY;

- national and international library consortia such as GASCO (Germany, Austria, Switzerland), INFER (Italy), COUPERIN (France), JISC (U.K.), ABM-uitvikling (Norway).

In the next months $\mathrm{SCOAP}^{3}$ aims to federate similar agents worldwide: the $\mathrm{SCOAP}^{3}$ model will only be successful if all countries contributing to the vast majority of the HEP literature become members of the consortium. Indeed, a pillar of the $\mathrm{SCOAP}^{3}$ model is to ensure OA to all HEP articles appearing in high-quality journals, irrespective of the affiliation of their authors. Manuscripts from authors without academic affiliation or authors from less-privileged countries, which cannot be reasonably expected to contribute to the consortium at this time, will be treated like all other articles. The ethical reason of conserving the access of any author to peer review is obvious. At the same time, this choice has solid financial reasons: restricting OA privileges only to authors affiliated to some countries would simply replace the present toll-access barriers with different barriers, connected to the geographical origin of the articles. Moreover, if only a geographical subset of the HEP scientific literature were available OA, consortium members would still be required to purchase the remaining fraction, with no evident financial benefits from the OA transition.

$\mathrm{SCOAP}^{3}$ will be financed with funds currently used for journal subscriptions by HEP funding agencies, laboratories and libraries. At the same time it will engage other bodies interested in the broad and free dissemination of scientific information. Each country will contribute to $\mathrm{SCOAP}^{3}$ in a "fair" way, according to its share of the worldwide HEP scientific production, as discussed in Section 5. For the $\mathrm{SCOAP}^{3}$ model to be successful, it should represent a stable, viable and sustainable alternative to subscriptions vis-à-vis its partners. It is therefore expected that the $\mathrm{SCOAP}^{3}$ operation will follow the financial blueprint of large HEP scientific collaborations, which usually bind over one hundred laboratories and universities in Memoranda of Understanding spanning several decades.

The innovation of the $\mathrm{SCOAP}^{3}$ model with respect to other OA options currently offered by most publishers is that it will centralize all OA expenses, which will not have to be borne by authors and research groups. These other "author-pays" options, of scarce success in HEP, are perceived as an even higher barrier than subscription charges, in particular for theoretical physicists from small institutions, whose articles account for the vast majority of HEP papers.

It is expected that $\mathrm{SCOAP}^{3}$ will contribute to stabilize the rising cost of access to information in the HEP domain by virtue of increasing author awareness to costs and prices, and by fostering new competition in the market, linking quality and price.

A large fraction of the publications on core HEP subjects is published in a limited number of journals [3], as detailed in sections 2 and 4. Among those journals, some carry almost entirely HEP content. SCOAP ${ }^{3}$ aims to assist publishers in converting these entire "core" journals to OA. It is expected that the vast majority of the $\mathrm{SCOAP}^{3}$ budget will be spent for "core" journals with a "lump-sum" payment: SCOAP" pays a negotiated price for the peer-review of all articles processed by a journal. Many HEP articles appear in "broadband" journals, which carry just a small fraction of HEP content. It is expected that these articles will be sponsored by SCOAP $^{3}$ on a "pay-per-article" basis. Conference proceedings and monographs are not within the scope of SCOAP".

In the $\mathrm{SCOAP}^{3} \mathrm{OA}$ model, the publishers will have the prime responsibility of ensure quality of the highest standards through independent editorial boards and the peer review. They will ensure the dissemination of OA articles by posting them onto their web sites and, in addition, feeding them to a SCOAP ${ }^{3}$ repository. 
Publishers will benefit from a more sustainable business model than the traditional subscription scheme, becoming increasingly fragile. They will continue to meet the demand for print subscription, re-print of single articles, color plates in these printed versions, collections of articles in electronic or paper form, citation databases and other "premium" services, which are outside the scope of SCOAP S $^{3}$

\section{The High Energy Physics Publishing Landscape}

The definition of HEP is often linked to the theoretical and experimental study of particles produced at accelerators of ever-increasing energy. Both the field and its definition have evolved to include subjects naturally more close to the fields of nuclear physics, of astrophysics and of cosmology. Different authors, different journals and different funding agencies each focus on different parts of the HEP spectrum and therefore have a different definition of the field.

To be successful, $\mathrm{SCOAP}^{3}$ should, at once, aim to convert to OA the subset of scientific literature of common interest to all players, while striving for as wide a scope as possible. A minimal set of common interest to the entire HEP community is constituted by a "core" set of topics such as the phenomenology and experimental investigations of elementary particles and their interactions, quantum-field theory and lattice-field theory. These topics are loosely related to the hep-ex, hep-lat, hep-ph, and hep-th areas of the arXiv.org repository, which often also carry content in cognate disciplines. Experimental techniques as well as mathematical and numerical methods are also included in this definition of HEP "core" articles. The definition of HEP article covers more loosely other fields of relevance to HEP, such as selected topics in nuclear physics, astrophysics, gravitation and cosmology.

It is important to note that the vast majority of HEP articles concern phenomenology and theory and have on average 2.6 authors [3]. On the other hand, publications on experimental results were often authored by up to 500 scientists in the last decade, while collaborations now publishing their analyses count up to 800 researchers and articles by LHC collaborations will have up to 2000 authors.

\begin{tabular}{|c|c|c|c|c|c|c|c|}
\hline $\begin{array}{r}\text { Journal } \\
\end{array}$ & Publisher & IF & $\mathbf{N}_{\text {tot }}$ & $\mathbf{N}_{\text {HEP }}$ & $\mathbf{N}_{\text {core }}$ & $\mathbf{f}_{\mathrm{HEP}}$ & $\mathbf{f}_{\text {core }}$ \\
\hline Phys. Rev. D & APS & 4.8 & 2285 & 2101 & 1635 & $92 \%$ & $72 \%$ \\
\hline JHEP & SISSA/IOP & 5.9 & 856 & 856 & 840 & $100 \%$ & $98 \%$ \\
\hline Phys. Lett. B & Elsevier & 5.3 & 957 & 862 & 740 & $90 \%$ & $77 \%$ \\
\hline Nucl. Phys. B & Elsevier & 5.5 & 522 & 481 & 465 & $92 \%$ & $89 \%$ \\
\hline Phys. Rev. Lett. & APS & 7.5 & 3836 & 407 & 279 & $11 \%$ & $7 \%$ \\
\hline Eur. Phys. J. C & Springer & 3.2 & 331 & 272 & 234 & $82 \%$ & $71 \%$ \\
\hline Mod. Phys. Lett. A & World Scientific & 1.2 & 281 & 216 & 138 & $77 \%$ & $49 \%$ \\
\hline Phys. Rev. $C$ & APS & 3.6 & 853 & 298 & 136 & $35 \%$ & $16 \%$ \\
\hline Class. Quant. Grav. & IOP & 2.9 & 491 & 255 & 89 & $52 \%$ & $18 \%$ \\
\hline Int. J. Mod. Phys. A & World Scientific & 1.5 & 878 & 143 & 88 & $16 \%$ & $10 \%$ \\
\hline J. Math. Phys. & AIP & 1.2 & 446 & 108 & 74 & $24 \%$ & $17 \%$ \\
\hline Phys. Atom. Nucl. & Springer & 0.9 & 220 & 106 & 72 & $48 \%$ & $33 \%$ \\
\hline$J C A P$ & SISSA/IOP & 6.7 & 156 & 128 & 57 & $82 \%$ & $37 \%$ \\
\hline Gen. Rel. Grav. & Springer & 1.6 & 190 & 103 & 20 & $54 \%$ & $11 \%$ \\
\hline Nucl. Instrum. Meth. A & Elsevier & 1.2 & 1371 & 312 & 16 & $23 \%$ & $1 \%$ \\
\hline
\end{tabular}

Table 1: The most popular HEP journals and their publishers, together with their ISI impact factor, IF; the total number of articles published in 2005, $N_{\text {tot }}$; the number of HEP articles, $N_{H E P}$; and the number of articles in the HEP core subject, $\mathbf{N}_{\text {core }}$. The journals are ordered in decreasing order of $\mathrm{N}_{\text {core }}$. Only journals with $\mathrm{N}_{\mathrm{HEP}}>\mathbf{1 0 0}$ are shown. The last two columns show the fractions $f_{H E P}$ and $f_{\text {core }}$ of $H E P$ and core articles, respectively. From reference [7].

In 2005, about 8'500 HEP articles were published in peer-reviewed journals, as included in the SPIRES database [9]. Of these, 5'200 articles are classified in the core HEP topics discussed above. Table 1 presents the most popular HEP journals and their corresponding publishers, together with their ISI impact factor, IF [10], and the total number of articles published in $2005, \mathrm{~N}_{\text {tot }}$. The number of HEP articles, $\mathrm{N}_{\mathrm{HEP}}$, is also listed together with the number of articles in the core subject areas of phenomenology and experimental investigations of elementary particles and their interactions, quantum-field theory and lattice-field theory, $\mathrm{N}_{\text {core }}$. The journals are ordered in 
decreasing order of $\mathrm{N}_{\text {core }}$. Only journals with $\mathrm{N}_{\mathrm{HEP}}>100$ are shown. The last two columns show the fractions $\mathrm{f}_{\mathrm{HEP}}$ and $\mathrm{f}_{\text {core }}$ of HEP and core articles, respectively [7].

As discussed in section 2, a recent study analyzed core HEP articles submitted in 2005 to the arXiv.org repository and classified in the hep-ex, hep-lat, hep-ph and hep-th categories and subsequently published. Out of a total of about 5'000 articles, more than $80 \%$ appeared in just six peer-reviewed journals from four publishers [3]. Five out of these six journals carry a majority of HEP content, as listed in table 1, these are:

- $\quad$ Physical Review D (published by the American Physical Society);

- $\quad$ Physics Letters B (Elsevier);

- Nuclear Physics B (Elsevier);

- Journal of High Energy Physics (SISSA/IOP);

- $\quad$ European Physical Journal C (Springer).

$\mathrm{SCOAP}^{3}$ aims to assist publishers in converting these "core" journals entirely to OA. As described in the last column of table 1, these five "core" journals include up to $30 \%$ of articles beyond the core HEP topics, particularly in Nuclear Physics and Astroparticle Physics. These articles will also be included in the OA conversion of the journals. This is in the interest of the HEP readership and promotes the long-term goal of an extension of the $\mathrm{SCOAP}^{3}$ model to these related disciplines.

The sixth journal, Physical Review Letters (American Physical Society), is a "broadband" journal, which carries only a small fraction $(10 \%)$ of HEP content. $\mathrm{SCOAP}^{3}$ aims to sponsor the conversion to OA of this fraction on an article-by-article basis. A similar approach holds for another popular "broadband" journal in instrumentation: Nuclear Instruments and Methods in Physics Research A (Elsevier), which carries about 25\% of HEP content.

These seven journals covered, in 2005, around 4'200 core HEP articles and about 5'300 articles in the wider HEP definition, including all related subjects. The conversion to OA of these five "core" journals and the HEP part of these two "broadband" journals would cover over $80 \%$ of the core HEP subjects and over $60 \%$ of the entire HEP literature, including all related subjects. The remaining 3'300 HEP articles, not published in the journals mentioned above, are scattered over some 140 other journals. It is important to note that the $\mathrm{SCOAP}^{3}$ model should not be limited to this set of journals but is open to all existing and future high-quality journals which carry HEP content, within budgetary limits.

\section{$5 \quad$ Financial Aspects of the SCOAP ${ }^{3}$ Model}

The price of a journal is driven by the costs to run the peer-review system, by editorial costs for copy-editing and typesetting, by the cost for electronic publishing and access control, and by subscription administration. Some publishers today quote a cost, from reception to final publication, in the range of 1'000 -2'000 Euros per published article [11]. This includes the cost of processing articles which are eventually rejected, the fraction of which varies substantially from journal to journal.

The annual budget for a transition of HEP publishing to OA can be estimated from this figure and the fact that the five "core" journals, which cover a large fraction of the HEP literature, publish about 5'000 articles per year. Hence, we estimate that the annual budget for a transition of HEP publishing to OA would amount to a maximum of 10 Million Euros per year.

Another indication which corroborates this estimate is that the costs to run a "core" journal such as Physical Review D, amount to 2.7 Million Euros per year [11] and it covers about a third of the HEP publication landscape [3].

A "fair-share" scenario for the financing of $\mathrm{SCOAP}^{3}$ is to distribute these costs among all countries active in HEP on a pro-rata basis, taking into account the size of the HEP author base of each country. To cover publications from scientists from developing countries, which cannot be reasonably expected to contribute to the consortium at this time, an allowance of not more than $10 \%$ of the $\mathrm{SCOAP}^{3}$ budget is foreseen.

The size of the HEP author base in each country is estimated from a recent study [7] which considered all articles published in the years 2005 and 2006 in the five HEP "core" journals, Physical Review D, Physics Letters B, Nuclear Physics B, Journal of High Energy Physics and the European Physical Journal C, as well as those HEP articles published in the two "broadband" journals, Physical Review Letters and Nuclear Instruments and Methods in Physics Research A. A total sample of about 11'300 articles was considered and, in each of them, all authors were uniquely assigned to a given country. CERN was treated as an additional country. 
In about $5 \%$ of the cases, authors were found to have multiple affiliations, often in different countries, reflecting the intense cross-border tradition of HEP. In these cases, the ambiguity in the assignment of authors to countries was solved as described in reference [7]. The results from this study are summarized in table 2 and figure 3.

\begin{tabular}{|l|r|}
\hline \multicolumn{1}{|c|}{ Country } & $\begin{array}{c}\text { Share of HEP } \\
\text { Scientific Publishing }\end{array}$ \\
\hline United States & $24.3 \%$ \\
\hline Germany & $9.1 \%$ \\
\hline Japan & $7.1 \%$ \\
\hline Italy & $6.9 \%$ \\
\hline United Kingdom & $6.6 \%$ \\
\hline China & $5.6 \%$ \\
\hline France & $3.8 \%$ \\
\hline Russia & $3.4 \%$ \\
\hline Spain & $3.1 \%$ \\
\hline Canada & $2.8 \%$ \\
\hline Brazil & $2.7 \%$ \\
\hline India & $2.7 \%$ \\
\hline CERN & $2.1 \%$ \\
\hline Korea & $1.8 \%$ \\
\hline Switzerland & $1.3 \%$ \\
\hline Poland & $1.3 \%$ \\
\hline Israel & $1.0 \%$ \\
\hline Iran & $0.9 \%$ \\
\hline Netherlands & $0.9 \%$ \\
\hline Portugal & $0.9 \%$ \\
\hline Taiwan & $0.7 \%$ \\
\hline Mexico & $0.5 \%$ \\
\hline Sweden & $0.8 \%$ \\
\hline Belgium & $0.8 \%$ \\
\hline Greece & $0.8 \%$ \\
\hline Denmark & $0.7 \%$ \\
\hline Australia & $0.7 \%$ \\
\hline Argentina & $0.6 \%$ \\
\hline Turkey & $0.6 \%$ \\
\hline Chile & $0.6 \%$ \\
\hline Austria & $0.6 \%$ \\
\hline Finland & $0.6 \%$ \\
\hline Hungary & $0.5 \%$ \\
\hline Remaining countries & $0.5 \%$ \\
\hline & \\
\hline
\end{tabular}

Table 2: Contribution to the HEP scientific publishing of several countries. Co-authorship is taken into account on a pro-rata basis, assigning fractions of each article to the countries in which the authors are affiliated. The last cell aggregates contributions from countries with a share below $0.4 \%$. This study is based on all articles published in the years 2005 and 2006 in the five HEP "core" journals, Physical Review

D, Physics Letters B, Nuclear Physics B, Journal of High Energy Physics and the European Physical Journal $C$ and the HEP articles published in two "broadband" journals, Physical Review Letters and Nuclear Instruments and Methods in Physics Research A. A total sample of about 11'300 articles is considered. From reference [7]. 


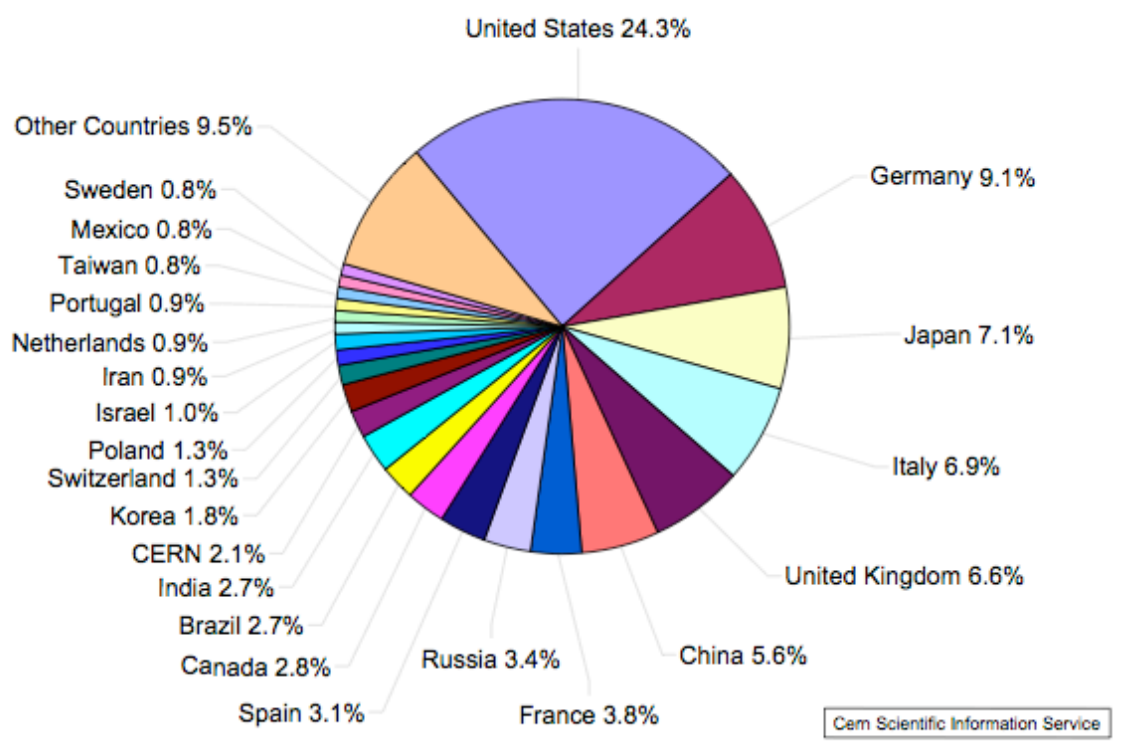

Figure 3: Contribution to the HEP scientific publishing of several countries. Co-authorship is taken into account on a pro-rata basis, assigning fractions of each article to the countries in which the authors are affiliated. The last cell aggregates contributions from countries with a share below $0.4 \%$. This study is based on all articles published in the years 2005 and 2006 in the five HEP "core" journals, Physical Review D, Physics Letters B, Nuclear Physics B, Journal of High Energy Physics and the European Physical Journal $C$ and the HEP articles published in two "broadband" journals, Physical Review Letters and

Nuclear Instruments and Methods in Physics Research A. A total sample of about 11'300 articles is considered. Contributions from countries with a share below $0.8 \%$ are summed in the slice denoted as "Other Countries". From reference [7].

\section{Conclusions and Outlook}

At the time of writing, $\mathrm{SCOAP}^{3}$ is an initiative emanating from leading European funding agencies, the two largest HEP European laboratories, and national and international library consortia. The fundamental pillar of the $\mathrm{SCOAP}^{3}$ model is the federation of HEP funding agencies and library consortia worldwide. HEP is the most global of the scientific enterprises and the conversion to OA of its literature, with all the ethical, scientific and financial benefits it implies can only be achieved in a global co-ordinated process. A crucial step towards OA publishing in HEP is therefore the search for a world-wide consensus around the SCOAP ${ }^{3}$ initiative, aiming to expressions of interest from HEP funding agencies and library consortia in Europe, the United States and beyond.

Once sufficient funds will have been pledged towards the establishment and the operation of $\mathrm{SCOAP}^{3}$, a tendering process involving publishers of high-quality HEP journals will take place. Provided that the SCOAP S $^{3}$ funding partners are ready to engage into long-term commitments, most publishers are expected to be ready to enter into negotiations along the lines presented in this article.

The outcome of the tendering process will allow the complete $\mathrm{SCOAP}^{3}$ budget envelope to be precisely known and therefore the precise contribution expected from each country. A Memorandum of Understanding for the governance of $\mathrm{SCOAP}^{3}$ will then be signed by funding agencies and leading national and international library consortia. Contracts with publishers will be established in order to make Open Access publishing in High Energy Physics a reality at the beginning of 2008, when the first experimental and theoretical publications of the CERN LHC program will appear.

The conversion of the HEP scientific publishing to the OA paradigm, along the lines presented in this article, will be an important milestone in the history of scientific publishing. The $\mathrm{SCOAP}^{3}$ model could be rapidly generalized to other disciplines and, in particular, to related fields such as Nuclear Physics or Astroparticle Physics. 


\section{Acknowledgements}

This work summarises the report of the SCOAP ${ }^{3}$ Working Party which, between December 2006 and April 2007 , drafted the blueprint for the establishment, financing and operation of the consortium, with vision and dedication. I am grateful to all members of the Working Party for having shared their unique experiences towards making OA in HEP a success: S. Bianco, O.-H. Ellestad, P. Ferreira, F. Friend, P. Gargiulo, R. Hanania, S. Henrot-Versille, A. Holtkamp, P. Igo-Kemenes, D. Jarroux-Declais, M. Jordão, B.-C. Kämper, J. Krause, T. Lagrange, F. Le Diberder, A. Lemasurier, A. Lengenfelder, C. Lindqvist, S. Plaszczynski, R. Schimmer, J. Vigen, R. Voss, M. Wilbers, J. Yeomas, K. Zioutas. We have been constantly inspired by the OA vision of CERN Director General, R. Aymar.

\section{Notes and References}

[1] http://oa.mpg.de/openaccess-berlin/berlindeclaration.html [Last visited 4 April 2007].

[2] VOSS, R., et al., Report of the Task Force on Open Access Publishing in Particle Physics, CERN, 2006; http://library.cern.ch/OATaskForce_public.pdf.

[3] MELE, S., et al., JHEP 12(2006)S01; arXiv:cs.DL/0611130.

[4] http://open-access.web.cern.ch/Open-Access/20050916.html [Last visited 4 April 2007].

[5] http://indico.cern.ch/conferenceDisplay.py?confId=482 [Last visited 4 April 2007].

[6] http://indico.cern.ch/conferenceDisplay.py?confId=7168 [Last visited 4 April 2007].

[7] BIANCO, S., et al., Report of the SCOAP $P^{3}$ Working Party, CERN, 2007; in preparation. To obtain a copy please contact Salvatore.Mele@cern.ch.

[8] http://www.soros.org/openaccess/read.shtml [Last visited 4 April 2007].

[9] http://slac.stanford.edu/spires [Last visited 4 April 2007].

[10] http://scientific.thomson.com/free/essays/journalcitationreports/impactfactor/ [Last visited 4 April 2007].

[11] BLUME, M. Round table discussion: Policy Options for the Scientific Publishing System in FP7 and the European Research Area. Conference on Scientific Publishing in the European Research Area: Access, Dissemination and Preservation in the Digital Age, Brussels 15-16 February 2007. 